\title{
Natural Selection and Thermodynamics of Biological Evolution
}

\author{
Georgi P. Gladyshev \\ International Academy of Creative Endeavors and N. N. Semenov Institute of Chemical Physics, Russian \\ Academy of Sciences, Moscow, Russia \\ Email: academy@creatacad.org
}

Received 14 February 2015; accepted 4 March 2015; published 9 March 2015

Copyright (C) 2015 by author and Scientific Research Publishing Inc.

This work is licensed under the Creative Commons Attribution International License (CC BY). http://creativecommons.org/licenses/by/4.0/

(c) (i) Open Access

\section{Abstract}

The author of this article proposes that the representation of Charles Darwin and Alfred Wallace's theory on "variation and selection" in the living world is a reflection of the action of hierarchical thermodynamics. Hierarchical thermodynamics is based on the law of temporal hierarchies and on the principle of substance stability. This principle enables the transmission of thermodynamic information between lower and higher structural hierarchies, in both forward and reverse direction: from nucleic acids to higher structural hierarchies and back. The principle of substance stability, in fact, is the main dynamical and thermodynamic mechanism of natural selection. It is alleged that the natural selection of atoms, molecules, organisms, populations, and other hierarchical structures takes place under the action of a variety of internal factors within organisms and the external environmental factors that can be considered as tropisms. Forms (design) of living organisms are formed as a result of spontaneous and non-spontaneous processes that lead to the adaptation of living systems to the environment. The selection is carried out as a result of the impacts of different energy types and the principle of substance stability at all levels of hierarchical structures. Actions of tropisms are presented by various members of the generalized Gibbs equation.

\section{Keywords}

Natural Selection, Hierarchical Thermodynamics, Principle of Substance Stability, The Origin of Life, Evolution

\section{Epigraphs}

"One of the principal objects of theoretical research in any department of knowledge is to find the point of view 
from which the subject appears in its greatest simplicity."

“... the true and only goal of science is to reveal unity rather than mechanism."

Henri Poincare

"Hierarchical thermodynamics in accordance with the laws of nature creates and optimizes forms and functions of living systems in their habitat. This optimization is connected with the search for minimums of specific Gibbs free energy formation of dynamic structures of all hierarchies.”

Author

\section{General Presentation}

This article is a brief overview of selected works of the author in the field of thermodynamics of the origin of life, biological evolution and aging of living beings. It should be noted that this area of research is the subject of many studies. For a long time, many scientists try to use thermodynamics to explain biological issues. Some of researchers have made achievements and progress, while the disagreements and arguments in this area do exist.

The review focuses on the theoretical issues concerning natural selection in biological evolution. Hierarchical thermodynamics of the author studies the complex thermodynamic systems, where some work aside from extension work is performed. It is alleged that living dynamic structures are the quasi-closed and quasi-equilibrium supramolecular systems. As a result of self-assembly, these structures are condensed and are converted into structures of higher hierarchies. It is shown that the variation of the chemical composition and the structure of living systems have thermodynamic orientation.

Representations of Charles Darwin and Alfred Wallace's theory on "variation and selection" in the living world is a reflection of the laws of hierarchical thermodynamics created by the author based on the solid foundation of J.W. Gibbs theory [1].

Hierarchical thermodynamics, using the principle of substance stability (the principle put forth by the author), describes evolution and development, and defines the appearance of living systems at all hierarchical levels. In a hierarchical thermodynamics introduced notion of dynamical thermodynamic mechanism of transformation of hierarchical structures [2]-[8]. Here we are talking about changes in the thermodynamic functions of states of systems in time. It is assumed that the functions of a state near to equilibrium systems have a real physical meaning [9], i.e., these features are similar to the functions of the state of relevant equilibrium systems. Thermodynamic theory of biological evolution is represented by the author, was published in leading journals, monographs, patents and essays notes on the Internet. It can be assumed that these works on thermodynamic theory of biological evolution are the foundations laid by the author, creating a new field of research that uses the methods of near-equilibrium thermodynamics. This new concept of thermodynamics uses representations of quasiclosed systems of variable composition. Dynamic behavior of these systems with a good approximation is described based on the representation of the functions of the state i.e., functions that have full differentials. In other words, it is assumed that biological systems at each hierarchical level can be characterized by functions of the states which have real physical meaning. This approach is consistent with the concept of non-equilibrium thermodynamics of systems close to equilibrium [9]. This approach highlights the difference between hierarchical thermodynamics and Prigogine's thermodynamics of systems far from equilibrium [10]. Prigogine's thermodynamics is actually a type of kinetics, because it cannot be described in terms of state functions. For instance, according to Prigogine's theory, the system's entropy has no full differential. Therefore, the idea of living dissipative structures cannot be considered thermodynamic.

Recently, Professor Fouad El-Diasty presented an ice original article on the "Origin of Order: Emergence and Evolution of Biological Organization as a Problem in Thermal Physics” [11]. However, the hierarchical thermodynamics of the origin of life and biological evolution (the variation and the selection that define evolution) describes the behavior of complex thermodynamic living systems, in which and over which various types of work can be performed [12]-[17]. This article of author takes into account this situation. Due to broad diversity within this research field which is beyond the scope of this review, we will consider only some general theoretical concepts. Of note, the author published many studies in peer-reviewed Russian scientific journals, which are not internationally known. References to these publications are generally not presented here. These references can be found in separate monographs and articles in leading journals, and the Internet. Various assumptions and approximations made while creating the thermodynamic theory of the origin of life, biological evolution and aging of living beings was considered in the author's works and cited in the bibliography. 


\section{History of the Application of Thermodynamics to the Problems of Origin of Life, Biological Evolution and Aging}

There are many attempts to apply thermodynamics for explaining the phenomena of life [8] [18]. L. Boltzmann wrote that life is connected with the production of negative entropy (negentropy). This concept was developed by E. Schrodinger in his famous work "What is life". In the future, I. Prigogine proposed to consider living objects as the far from equilibrium "dissipative structures”. He argued that the thermodynamics of J. W. Gibbs [1] is of no interest from the perspective of the origin of life, evolution and aging of living beings. Moreover, long before the mentioned works of I. Prigogine, Erwin Bauer introduced the concept of "structural energy" and formulated the principle of sustainable disequilibrium: "All and only living systems are never in equilibrium and perform due to its free energy constantly working against the equilibrium...” However, Bauer's theory was not experimentally confirmed. It is difficult to understand the origin of "work against the equilibrium”. One cannot say what kind of equilibrium, chemical or supramolecular, was investigated in these works. Furthermore, we now know that the independent local supramolecular and other quasi-equilibriums do exist in living systems.

"Thermodynamics of dissipative structures" is certain kinetics describing the behavior of systems far from equilibrium. In this “thermodynamics” there are no state functions. For example, "Prigogine’s entropy” in the system is far from equilibrium and has no full differential. This "entropy” cannot be calculated or determined [15]-[17]. Prigogine's theory, at best, can be regarded as a trial or deadlock theory. Representations of Prigogine's theory were widely used by M. Volkenstein, A. Zotin and many of their followers. However, these researchers were eclectic as they often mixed some concepts of Prigogine's theory with that of J.W. Gibbs theory and the "information entropy" of K. Shannon. These scientists and a number of gerontologists apparently were far from the modern ideas on the subject. In fact, my opinion on the quasi-equilibrium hierarchical thermodynamics of biological evolution and aging is that the works of Prigogine and his colleagues are now of historical interest only.

The author of this article believes that it is hardly appropriate to use clearly unfounded statements of L. Boltzmann, E. Schrodinger, I. Prigogine and other researchers that associate the phenomenon of life and its evolution with an increase in the entropy of the universe (or negentropy of living systems) only. A huge amount of mistakes exist due to the unacceptable confusion in terminology and a lack of understanding or neglect by many authors on the foundations of equilibrium thermodynamics, as well as quasi-equilibrium hierarchical thermodynamics applicable to describe the evolution of living and non-living matter. For example, the conclusions on the thermodynamics of ideal systems are being extrapolated to "the complex systems with interactions" or reduce everything to the change of "imaginary entropy" [18] [19].

It is noteworthy that a number of scientists long before I. Prigogine realized the "spirit and letter" of classical thermodynamics by Clausius-Gibbs and put forward reasonable concepts of the origin of life and its evolution from the viewpoint of physical chemistry. It is necessary to point out the well-known outstanding works of Alexander Oparin, John Haldane, Stanley Miller and Harold Urey. Currently, many organic chemists and physical chemists are studying the origin of life at the molecular level based on classical thermodynamics. Here we can mention the outstanding works of David Dimler and his colleagues, who offer a very reasonable model of the origin of life. Proceedings of these scientists are posted online. In recent decades, researchers have begun to pay attention to the role of catalysis in the phenomena of life. Many important considerations are presented from the perspective of the kinetics and mechanisms of these phenomena.

The author presented the history of hierarchical thermodynamics in [8] and in essays online. It was noted that the open nature of biological systems is not an obstacle for using the same methods of thermodynamics to study them. It is appropriate to recall the methods of equilibrium chromatography to study the dynamical systems in the case of quasiequilibrium [7].

Access to the Internet has triggered widely distributed controversies regarding ideas about life and its origins. For example, the online multi-volume edition EoHT [19] discussed statements belonging to not only professional researchers, but also to great scientists from other subject areas, who are specialists in their own fields. In addition, opinions of science enthusiasts and lay persons on the problems of the origin of life and its evolution are also presented. But in some cases, many issues are described from perspectives that are not consistent with modern science. For example, there are discussions of the views concerning "the defunct theory of life” [20] and the idea about "molecules" as different types of particles and bodies of different nature in the EoHT [19]. One table in EoHT shows the experimental data on the atomic composition of organisms. However, I believe the au- 
thor of this article in EoHT clearly used an unacceptable concept of molecules. In addition, as in the past century, "Human thermodynamics" is often considered as thermodynamics of simple systems (ideal gases or ideal solutions). However, thermodynamics of interaction between people (as well as any organisms) must be investigated from the perspective of complex systems that perform various kinds of work, not just the work of expansion [4] [5] [17]. The categorical rejection by some authors that strictly warranted established views on the problems of life is not conducive to the realization of the progress in this field of research. Nevertheless, I note that the above-mentioned work may be useful to historians of science and art, which distinguish fantasies from reliable information.

\section{The Hierarchical Approach to the Study of Life and the Law of Temporal Hierarchies}

The study of life, as a phenomenon is greatly simplified if we consider the processes in living systems as a transformation of structure of living objects. This transformation leads to the appearance of well-known hierarchical systems, which arise due to the condensation of the structures of each of the lower hierarchy to form the structures of the higher hierarchies [2] [5]-[7]. This sequential condensation is a "structural collapse of living matter”, which consistently unites molecules, supramolecular structures, cells, organisms, populations and other structures resulting from the self-assembly. The collapse of living matter develops under the action of hierarchical thermodynamics and this process is a reverse process of branched chain reaction. In a limited sense, it resembles the gravitational collapse. Inside each hierarchy this collapse is similar to the crystallization of individual substance which is accompanied by the formation of a new phase. From this perspective, the development of a living system is a hierarchical process of phase formations. Hierarchical thermodynamics was created on the basis of Gibbs's thermodynamics [1], which is the most accurate physical theory. Hierarchical thermodynamics allow us in quasi-approximation to explain the known phenomena and to make numerous predictions.

Hierarchical thermodynamics was created from the discovery of the law of temporal hierarchies, which proves the possibility of an independent study of the processes within each hierarchical level [2] [5] [7].

The law of temporal hierarchies was formulated by the author in the late 70s of the XX century [2]. Some colleagues referred to the law as "Gladyshev's law" or "Gladyshev law". One of the formulations of this law can be defined as: the law of temporal hierarchies states that any living system of any temporal hierarchical level in a normal state has a thermostat (physical thermostat), which is a surrounding medium that is characterized by slightly changing average values of thermodynamic parameters [3] [5]. In simple terms, the law of temporal hierarchies is a means of identifying or discerning a systems-within-systems point of view in the classical thermodynamic analysis of a living system both internally and socially. The main reason for this statement is the connection with the phenomenon of metabolism. Lower level hierarchical structures are often reproduced in a medium of higher-level hierarchical structures during the lifetime of the latter. Thus, we have:

$$
t^{j} \ll t^{j+1}
$$

where $t^{j}$-average lifetime of structures of lower hierarchical level, $t^{j+x}$ —average lifetime of structures of higher hierarchical level.

I would like to stress that the law of temporal hierarchies makes it possible to identify quasi-closed thermodynamic systems (subsystems) in open biosystems [3] [5]. It is possible to study their development (ontogenesis) and evolution (phylogenies) by studying the changes of the value of specific (per unit of volume or mass) Gibbs function of the formation of the given higher hierarchical structure out of lower-level structures. Thus, it was established that in ontogenesis (or phylogenies), the specific Gibbs function of the formation of supramolecular structures of an organism's tissues $\left(\overline{\tilde{G}}_{i}^{i m}\right)$ tends to be a minimum:

$$
\overline{\tilde{G}}_{i}^{i m}=\frac{1}{V} \int_{0}^{V} \frac{\partial \overline{\tilde{G}}_{i}^{i m}}{\partial m}(x, y, z) \mathrm{d} x \mathrm{~d} y \mathrm{~d} z \rightarrow \min
$$

where $V$ is the volume of the system; $\underline{m}$ is the mass of the identified micro-volumes; $x, y, z$ are coordinates; symbol “-” means that the value $\overline{\tilde{G}}_{i}^{i m}$ is specific; and symbol “ " emphasizes the heterogeneous character of the system. Let us note that Equation (2) implies taking account of all supramolecular interactions in all hierarchical bio-tissue structures (intracellular, intercellular and others). This is fully justified because the structural 
hierarchy does not always coincide with the temporal hierarchy. Thus, some types of cells do not divide and like organisms, age simultaneously with the organism. However, any supramolecular hierarchy $(j-1)$ has some higher hierarchy $(j+x)$, so that

$$
t^{j-1} \ll t^{j+x}
$$

where $t^{j-1}$ and $t^{j+x}$ are the mean duration of the existence of the corresponding structural hierarchies in a living system, and $x=0,1,2, \cdots$, etc.

The use of equality (2) means, in fact, that we apply the law of temporal hierarchies as:

$$
\cdots \ll t^{m} \ll t^{i m} \ll t^{\text {organism }} \ll t^{\text {pop }} \ll \cdots
$$

Here, $t^{m}\left(t^{c h}\right)$ is the average life span of an organism's molecules (chemical compounds) that take part in metabolism, $t^{i m}\left(t^{\text {supra }}\right)$ is the average life span of any supramolecular structures of an organism's tissues that are renewed in the process of its growth and development, $t^{\text {organism }}$ is the average life span of an organism in a population and $t^{\mathrm{pop}}$ is the population's average life span. The series of strong inequalities (3) does not include the life span (lifetime) of cells (cell) and some other supramolecular structures. However, this series of course tallies well with reality and reflects the existence of temporal hierarchies in living systems. The latter rigidly substantiates the possibility of identifying quasi-closed systems (subsystems) in open biological systems.

The thermodynamic theory of biological evolution and the aging of living beings accords with numerous facts and with mankind's empirical experience.

The existence of laws 1 and 3 allows us to use quasi-closed thermodynamic models to investigate living systems. I note that the sequences (1) and (3) are the series of overlapping triads of Nikolai Bogolubov [21]. This clearly proves the possibility of using thermodynamics for describing each hierarchical level.

\section{The Principle of Substance Stability. Biological Diversity and Natural Selection}

In hierarchical thermodynamics, the principle of substance stability describes the tendency of natural systems to seek local and general equilibria at all temporal and structural levels of organizational matter [5]. For example, it establishes a predominant direction of stable and unstable atomic and molecular movements between hierarchies. The principle of substance stability was presented in the form of a figure in a preprint in 1977 and in the paper published in 1978 [2]. Laterit was formulated as "the principle of the stability of a chemical substance" in the books published in 1996 and 1997 [5] [7]. This principle is now applied to all hierarchies [22]-[25]. The author called this principle as "the principle of substance stability" [25]. This principle in fact, is the main "thermodynamic mechanism" of natural selection. In a concise form, "the principle of substance stability" states that:

"During the formation or self-assembly of the most thermodynamically stable structures at the highest hierarchical level $(j)$, e.g., the supramolecular level, nature in accordance with the second law, spontaneously and predominantly uses the least thermodynamically stable structures available from a given local part of the biological system, belonging to a lower level, i.e., the molecular level $(j-1)$. Moreover, nature incorporates these unstable structures into the next higher level i.e., the supramolecular level $(j)$.”

In short, the principle of substance stability argues that each subsystem of the biosphere evolves according to its thermodynamic tendency to seek free energy minimums during each evolution cycle.

Note that in 1977 the author wrote "after the concluding stages of general evolution the concentration of free energy occurs not only owing to the growth of the chemical component $G j$-im (intermolecular) and higher order components, but also owing to the $G-j$ at the atomic component (as well as to other components not considered here)". In these early formulations of the principle of substance stability based on hierarchical thermodynamics, I believed in the importance of the atomic component ( $G j$-at) for understanding the direction of biological evolution. Laterit was showed that the composition variation (the selection) of chemical elements (atoms) during evolution indeed corresponds to "the principle of substance stability" [19] [26]. The same situation should be found with the variation of isotope concentration of elements during ontogenesis and phylogenies. This prediction was confirmed in the future [27]. In the sphere of sociology, with reference to family ties and their relation to society, the principle of substance stability operates in social hierarchies [17]. In a generalized social matrix one can visualize the substance considered as an elementary structure of any "inside" social hierarchy being defined as an understructure hierarchy, for e.g. a hierarchy of organisms, groups of organisms, etc. To cite one 
example, the stronger the love and mutual understanding between the husband and the wife, i.e., the understructure hierarchy, the less time they spend “outside” the family, i.e., the "over-structure hierarchy”. Marriage partners in types of stable relationships will not have the desire or impetus to seek external interaction and bonding as compared to less stable couples. Furthermore, the principle of substance stability corresponds to the wellknown rules of maintenance of stabilities of parties, unions, states, and nations, as personified by the age-old social management custom of “divide and rule”, in which higher hierarchies have fewer but more powerful and stable members [17]. The levels of the Gibbs function of formation of all monohierarchical systems during the emergence and degradation of living matter shift to lesser negative values. It should be noted that in his works the author wrote primarily about natural selection with common positions of hierarchical thermodynamics. In some articles he discusses only the general thermodynamic mechanisms of natural selection. Undoubtedly, in the future is interesting to study the important details of these processes.

In line with the presence of feedbacks among the structures in different hierarchies, this scheme should be presented as [2] [3] [5] [16] [17]:

Molecular structures (nucleic acids-proteins, other biological polymers and molecules) $\rightleftarrows$ Supramolecular structures $\rightleftarrows$ Cells $\rightleftarrows$ Organisms $\rightleftarrows$ Populations $\rightleftarrows$ Communities $\rightleftarrows$ Ecosystems, etc.

Arrows in this scheme $\rightleftarrows$ indicate the possibility of reading off direct and reverse (backward) information. As was repeatedly stressed, the rate of transmission of reverse information is, for the examined reasons, low as compared with that of direct information. This scheme (in accordance with the well-known facts) points to the relatively slow effect of the environment on the structure and characteristics of populations (on the time scale of their life) and in the final analysis, on the supramolecular and chemical structure of nucleic acids.

Figure 1 presents the scheme of the change in the Gibbs function (the Gibbs free energy of formation of structures of the biological world). This scheme is a scheme of a cycle of the relative circulation of matter in nature. This cycle can be studied from the viewpoint of hierarchical thermodynamics [18] [22] [25].

The long arrows in the center (the gray area) of the scheme at the bottom show the direction of thermodynamic self-organization (self-assembly) of the elementary structures of every $(j-1)^{\text {th }}$ monohierarchy, which are condensed to form the structures of the next (higher) $j^{\text {th }}$ monohierarchy. The short parallel arrows of the scheme show the displacement of Gibbs function to the back side- to lesser negative values during ontogenesis, phylogenies and evolution. This, as was already noted, is a consequence of the effect of the principle of substance stability! The principle contributes to the natural selection at each hierarchical level.

The bottom of Figure 1 shows the relatively stable chemical substances including minerals. These relatively stable compounds under the influence of different types of energy (in conditions of the existence of planets and other celestial bodies, or in space) transform to unstable products or products with low stability (top of Figure 1). Such products are $\mathrm{C}_{2} \mathrm{~N}_{2}, \mathrm{HCN}, \mathrm{C}_{5} \mathrm{H}_{5} \mathrm{~N}_{5}$ (adenine) among others [24] [25].

Of course the stability of these substances is determined by the conditions of their formation and existence. Furthermore, a comparison of the stability of the substances (for example, under standard conditions) is relative, because the stability of all the elements (simple substances) is equal to zero (i.e., the stability is accepted to be the same for all elements). However, the identification of new thermodynamic correlations in the "chemistry of life" and the confirmation of numerous predictions shows that this comparison is justified and reasonable. One rationale for this is the author's argument that the basic elements of life, apparently, can be considered as "objects of same type”. Of course the foregoing statement must be subjected to an additional test.

In conditions of the earth, the conversion to relatively stable chemicals occurs under the influence of solar and other energies. As a result of this exposure, sugars, amino acids and other products that are involved in the spontaneous chemical reactions and processes of hierarchical supramolecular condensation are formed.

It should be kept in mind that the energy of the external environment acts in all hierarchical structures of the living matter. This leads to the transformation (the conversion) of structures of all hierarchical levels and their mutual adaptation. Due to the large variety of environmental conditions (i.e., the surrounding environments of all hierarchies of living structures), there is a wide variety of structures in all hierarchies. The intensity of the action of physical fields and forces to different hierarchical structures of living matter is determined by the nature of these structures and, above all by their sizes [5] [25]. For example, light mainly contributes to molecular transformations but mechanical and gravitational fields are manifested primarily at the level of organisms. In general, the series of transformation of living matter involves electromagnetic forces, thermal effects (thermal energy), acoustic, mechanical, gravitational and all other possible actions of the surrounding environment. All of these effects can be represented as tropisms, which manifest themselves in the form of behavioral responses of living bodies or their adaptation to the environment [18] [25] [28] [29]. 


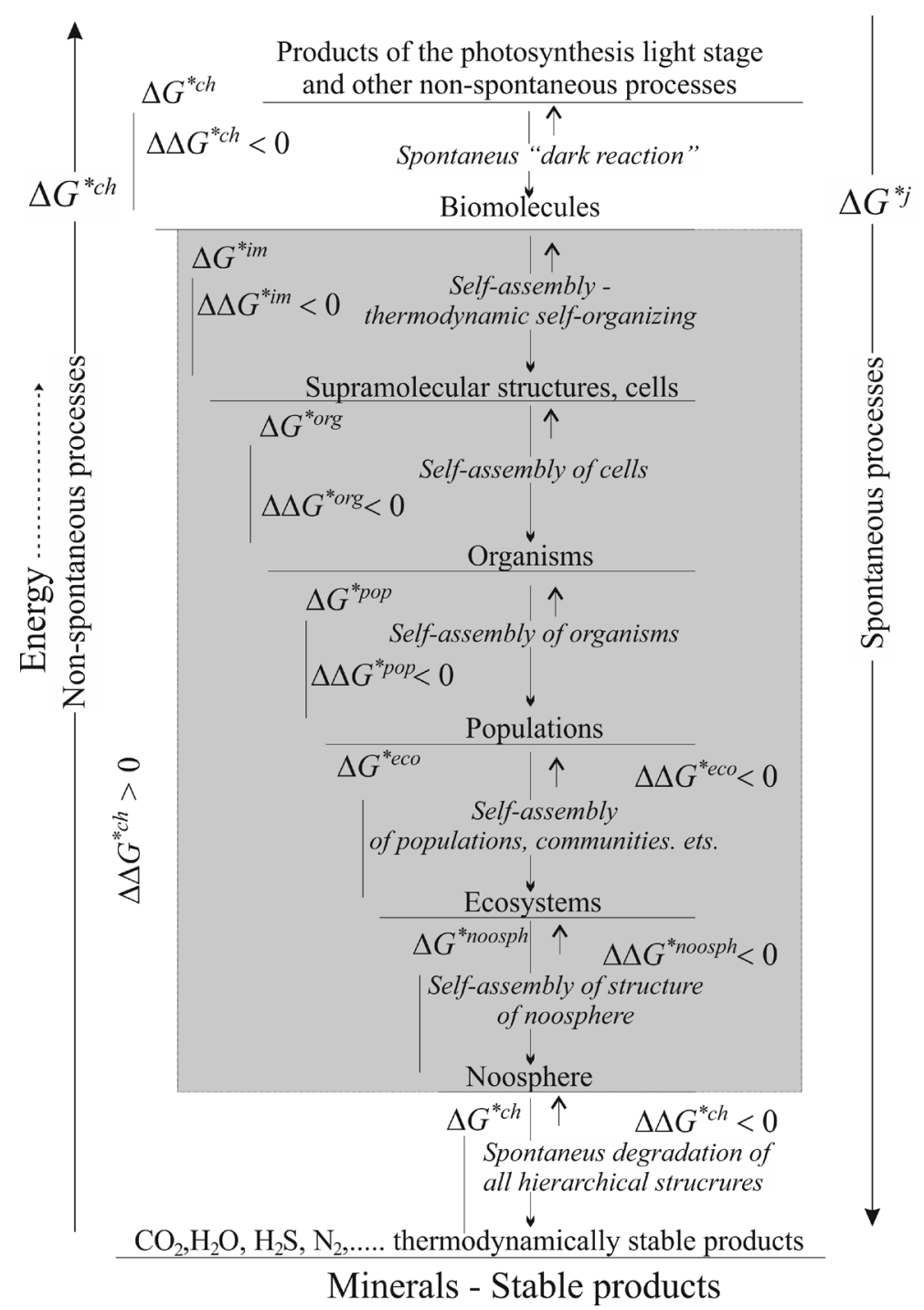

\footnotetext{
Figure 1. Scheme of the change in the Gibbs function of formation of complex thermodynamic systems $\Delta G^{* I}$ during the emergence and degradation of chemical (ch) and supramolecular structures (im), as well as organisms (organism), populations (pop), communities (com), ecosystems (eco), and the noosphere (noosph). The index $j$ is the type of structural hierarchy.
}

Thus, a variety of environmental conditions lead to high biological diversity and selection of living beings in a constantly changing environment. It is clear that on earth there are so many different places of dwelling for living beings. In other words, there are a myriad of physical thermostats [18] [25] [26], inhabited by a variety of organisms. Although the parameters of such thermostats are constantly changing, we can reasonably assume that during the relatively small time of existence of these thermostats, living structures have time to adapt to their environment. In brief, living systems (organisms) exist in dynamic quasi-equilibrium conditions. Supramolecular and higher quasi-equilibriums can be adapted to the environment during a short time. Environmental changes due to the action of the principle of substance stability can be recorded in the structure of nucleic acids-the carriers of hereditary information. This promotes natural selection. However, when revolutionary changes take place (e.g. action of volcanic eruptions, falls of meteorites, and other disasters), living systems cannot adapt to dramatically changed environment and they die. Thus, natural selection is only possible in the case of evolutionary processes when the selection thermodynamics is active.

All of the above is true for artificial selection, which is implemented within individual species of living beings but hampered in the interaction of different species. 
Natural selection and adaptation can occur at the social level of development of human society. Thus, beautiful and talented people congregate in large cities and developed countries where there are good living conditions and work in various spheres of human activity. There are many analogical examples, some of which are presented by the author in [17] [30] [31].

In the study of evolutionary transformations in living systems including natural selection, for pre-qualitative assessment of the role of individual processes in evolution, one can consider the well-known equation for the total differential of the Gibbs function [5] [7] [25]:

$$
\mathrm{d} G^{*}=\sum_{i} \mathrm{~d} G_{i}^{*}=-\sum_{i} S_{i} \mathrm{~d} T_{i}+\sum_{i} V_{i} \mathrm{~d} p_{i}-\sum_{i} \sum_{k_{i}} x_{k_{i}} \mathrm{~d} X_{k_{i}}+\sum_{i} \sum_{k_{i}} \mu_{k_{i}} \mathrm{~d} m_{k_{i}}
$$

where: $G$-Gibbs free energy; $T$-temperature; $S$-entropy; $V$-volume; $p$-pressure; $X$-any generalized force except pressure; $x$-any generalized coordinate except volume; $\mu$-chemical (evolutionary) potential; $m$-mass of $k$-substance; work realized by the system is negative. Index $i$ pertains to the specific evolution, $k$-to the component $i$ evolution. The superscript ${ }^{*}$ means that the behavior of a quasi-equilibrium complex system is considered.

The above equation is a generalized equation since in principle all interactions (inside and outside) of all structures of every hierarchical level are taken into consideration independently of the scale of these interactions. It is logical to consider this equation as one with considerably divided parameters, symbolic or speculative, that can be efficiently used only in relation to everyone or adjacent hierarchies of structures.

In this case, the Gibbs equation is considerably simplified in connection with negligibly small values of the majority of its isolated or individual members. Symbolism or speculation consists in the fact that it is difficult to take into consideration simultaneously all multi-scale effects determining the behavior of complex heterogeneous poly-hierarchical system at once. This statement is connected to the principle of mathematics, which allows us to combine like with like only and prefers the simplicity and unity in the description of the physical picture of the real world. Although, I should note that the above equation is characterized by a unity that is associated with a general thermodynamic approach to study the behavior of all individual mono hierarchical systems that form polyhierarchical systems.

Importantly, the simplified relations obtained from Equation (4) are used in the study of tropisms [10] [18] [25] [28] [29] and in many physiological and other studies [29]. The law of temporal hierarchies first substantiated the correctness of these approaches based on thermodynamics.

It should also be noted that the present equation must contain terms that take into account the interaction between biological objects (e.g. cells, organisms and other structures) arising from actions, physical fields and radiation, the origin of which is associated with chemical and physiochemical processes in living systems. Many that are often not strictly identified as "weak" interactions in the living world can be attributed to tropisms, which detail the mechanisms of these interactions, are not clear. The complexity of studying these phenomena, as a rule, is the lack of highly sensitive physical devices that measure these interactions. However, biological sensors can detect these mutual effects of biological objects. Thus it can be argued that the transfer of "structure information” between biological molecules and supramolecular structures by organisms do not necessarily require direct close contact of the said structures (e.g. information transfer that occurs at the contact of nucleotides during the formation of DNA or during the formation of acetic acid dimers).

Presented in this section, the data suggest that it is difficult to give a general definition to the phenomenon of life [20]. The only thing in common is that the characteristic of life apparently is a hierarchical structure and development of living objects. There are many definitions of life from different perspectives. One of the definitions of life based on thermodynamics is: the existence of spatially allocated polyhierarchical renewing structures in the circulation (in the cycle) of labile chemicals in the presence of liquid water on the planet.

\section{Final Remarks on the Predictions of Hierarchical Thermodynamics}

Hierarchical thermodynamics, despite a number of approximations, can explain many known facts and make a number of predictions. The author is not aware of any events and facts in the life sciences, which cannot be understood at least in principle, from the perspective of this field of science.

In 1977 it was shown that the variation of chemical and also supramolecular composition of living beings is a result of the action of laws of hierarchical thermodynamics [2] [5] [7]. The theory predicted enrichment of living beings by heavy isotopes and heavy chemical elements during evolution [27]. The author of this article unders- 
tood the nature of molecular selection in evolution including the selection of lipids, proteins and nucleic acids from the viewpoint of physical chemistry [3]. It was termed as thermodynamic dietetics [25] [32] [33]. On the basis of calculations presented, some recommendations were made regarding the establishment of anti-aging diets prolonging healthy life of animals and humans [25] [32]-[34]. These recommendations are fully consistent with the experience of ancient and modern medicine, and nutritional science. Furthermore, the author has made a number of completely new, practically important, recommendations in sport medicine, pharmacology and other areas of expertise [3] [7] [25]. It can be argued that through the principle of substance stability [25], hierarchical thermodynamics determines the natural selection of elements, molecules and structures of the higher hierarchies during the origin of life and biological evolution. This area of science has accumulated a lot of important information [26] [35], which should be carefully analyzed from the standpoint of thermodynamic selection.

Hierarchical thermodynamics allows us to understand social phenomena. From the standpoint of thermodynamics we can understand the ancient principle of "divide and rule". It is also shown that for most times the history of the development of society is predictable [16] [21].

On the basis of thermodynamic theory, hypotheses on the origins of cancer, the nature of some pathology, the common (identical) genetic code in the universe, and about the phenomenon of life as self-defending process can also be formulated [18] [27].

In recent years, attention was drawn to the opportunity to explain the phenomenon of natural selection based on thermodynamic concepts of the dynamical mechanism of this phenomenon. The author of this article every time emphasizes that the thermodynamics of biological phenomenon should not be looked into independent. This phenomenon has to be considered together with the thermodynamics of external environment.

\section{Conclusion}

The presented results give reason to believe that natural selection structures at all levels of organization of chemical and biological matter are the result of the laws of thermodynamics, and above all the principles of substance stability. The principle of substance stability reflects the basic thermodynamic mechanism of natural selection. From the perspective of this principle, we can explain the origin of life and its evolution, development and aging of living systems at all levels of biological organization. It can be assumed that hierarchical thermodynamics is the driving force behind the emergence and development of life in the universe.

\section{References}

[1] Gibbs, J.W. (1876) On the Equilibrium of Heterogeneous Substances. Vol. 1, Thermodynamics, Ox Bow Press, Connecticut.

[2] Gladyshev, G.P. (1978) On the Thermodynamics of Biological Evolution. Journal of Theoretical Biology, 75, $425-441$. http://dx.doi.org/10.1016/0022-5193(78)90354-5

[3] Гладышев, Г.П. (1988) Термодинамикаимакрокинетикаприродныхиерархическихпроцессов, Наука, Москва.

[4] Гладышев, Г.П. (1995) Термодинамика иерархических систем. В: Зефиров Н.С., Ред., Химическая энциклопедия, 4, Большая Российская Энциклопедия, Москва, 1062. http://www.xumuk.ru/encyklopedia/2/4371.html

[5] Gladyshev, G.P. (1997) Thermodynamics Theory of the Evolution of Living Beings. Nova Science Publishers, Inc., Commack, New York.

[6] Gladyshev, G.P. (1994) A Motive Force of Biological Evolution. Herald of Russian Academy of Science, 64, 118.

[7] Гладышев, Г.П. (2003) Супрамолекулярная термодинамика-Ключ к осознанию явления жизни (Что такое жизнь с точки зрения физико-химика), Издание второе, Институт компьютерных исследований, МоскваИжевск.

[8] Гладышев, Г.П. (2004) Об истории создания термодинамической теории происхождения жизни, биологической эволюции и старения живых существ. Журнал «История науки и техники», 1, 28. http://gladyshevevolution.wordpress.com/

[9] Denbigh, K.G. (1953) Thermodynamics of the Steady State. Methuen, London.

[10] Prigogine, I. (1980) From Being to Becoming: Time and Complexity in the Physical Sciences. W. H. Freeman and Company, San Francisco.

[11] El-Diasty, F. (2011) Origin of Order: Emergence and Evolution of Biological Organization as a Problem in Thermal Physics. Advances in Life Sciences, 1, 30-39. http://article.sapub.org/10.5923.j.als.20110101.06.html

[12] Sychev, V.V. (1973) Complex Thermodynamic Systems. Consultants Bureau, New York and London. 
http://dx.doi.org/10.1007/978-1-4684-1605-3

[13] Sychev, V.V. (1986) Thermodynamics of Complex Systems. Energoatomizdat, Moscow.

[14] Сычев, В.В. (2009) Сложные термодинамические системы. 5-е Издание, Издательский дом МЭИ, Москва.

[15] Gladyshev, G.P. (2003) Thermodynamic Self-Organization as a Mechanism of Hierarchical Structures Formation of Biological Matter. Progress in Reaction Kinetics and Mechanism, 28, 157-188. http://dx.doi.org/10.3184/007967403103165495

[16] Gladyshev, G.P. (2004) Macrothermodynamics of Biological Evolution: Aging of Living Beings. International Journal of Modern Physics B, 18, 801-825. http://dx.doi.org/10.1142/S0217979204023970

[17] Gladyshev, G.P. (2007) Leonhard Euler’s Methods and Ideas Live in the Thermodynamic Hierarchical Theory of Biological Evolution. International Journal of Applied Mathematics and Statistics, 11, 52-68.

[18] Гладышев, Г.П. (2014) Термодинамикавозникновенияжизни, эволюцииистарения. Успехигеронтол., 27, 225228.

[19] Thims, L., Ed. (2014) EoHT. http://www.eoht.info/

[20] Gladyshev, G. (2014) Life as a Phenomenon. International Journal of Applied Life Sciences and Engineering, 1, 97-98. http://www.ijalse.org/Volume 1Issue 1

[21] Bogolubov, N.N. (1990) Selected Works, Part 1, Dynamical Theory. Gordon and Breach Science Publishers, New York.

[22] Gladyshev, G.P. (2006) The Principle of Substance Stability Is Applicable to All Levels of Organization of Living Matter. International Journal of Molecular Sciences, 7, 98-110. http://www.mdpi.org/ijms/papers/i7030098.pdf http://dx.doi.org/10.3390/i7030098

[23] Gladyshev, G.P. (1999) On the Thermodynamics, Entropy and Evolution of Biological Systems: What Is Life from a Physical Chemist's Viewpoint? Entropy, 1, 9-20. http://www.mdpi.com/1099-4300/1/2

[24] Gladyshev, G.P. (2014) Life as a Process of the Existence, Reproduction, and Aging of Polyhierarchical Systems. Advances in Gerontology, 4, 1-2. http://dx.doi.org/10.1134/S2079057014010032

[25] Gladyshev, G.P. (2014) The Thermodynamic Theory of Evolution and Ageing. Advances in Gerontology, 4, $109-118$. http://dx.doi.org/10.1134/S2079057014020064

[26] Sterner, R.W. and Elser, J.J. (2002) Ecological Stoichiometry: The Biology of Elements from Molecules to the Biosphere. Princeton University Press, Princeton.

[27] Gladyshev, G.P. (2014) On the Change in the Isotope Compositions of Living Organisms during Aging and Evolution. Advances in Gerontology, 4, 107-108.

[28] Гладышев, Г.П. (2013) Тропизм как общее явление во вселенной. Можно ли поведение систем различной природы описать «Единой формулой»? Международный симпозиум «Современные проблемы высшего образования и науки в области химии и химической инженерии», Издательство Казахского Национального Университета, Алматы, 30-31 мая 2013, 7-11.

[29] Gladyshev, G. (2014) Thermodynamics Optimizes the Physiology of Life. Philosophy \& Cosmology, 12, 152-162. http://ispcjournal.org/journals/2014/PhC_2014.pdf

[30] Гладышев, Г.П. (2014) Дизайнитермодинамика. Международный творческий проект «ИСКУССТВО-ПРИРОДАСПОРТ», Российская Академия художеств, Москва, 23-25 января 2014.

[31] Gladyshev, G.P. (2014) Иерархическая термодинамика формирует дизайн окружающего мира. http://gladyshevevolution.wordpress.com/

[32] Gladyshev, G.P. (2007) The Thermodynamic Theory of Aging in Action: Medical Nutrition Recommendations for Patients of Any Age. In: Klats, R. and Goldman, R., Eds., Anti-Aging Therapeutics, Volume IX, American Academy of Anti-Aging Medicine, Chicago, 135-152.

[33] Gladyshev, G.P. (2007) Hierarchical Thermodynamics-General Theory of Existence and Living World Development: Model of Aging and Anti-Aging Quality of Foods and Medicines. Proceedings of the 15th Annual World Congress on Anti-Aging Medicine \& Regenerative Biomedical Technologies, Las Vegas, 12-15 December 2007. http://www.prolibraries.com/a4m/?select=session\&sessionID=1152

[34] Gladyshev, G.P. (1999) Method for Measuring the Gerontological Value of Biologically Active Substances and Compositions, Mainly Food and Cosmetic Products. Canadian Patent No. 2327747.

[35] Williams, R.J.P. and Frausto da Silva, J.J.R. (1997) The Natural Selection of the Chemical Elements: The Environment and Life's Chemistry. Clarendon Press, Oxford. 